\title{
GENERAL CONNECTIONS ON VECTOR BUNDLES
}

\author{
By NAOTO ABE
}

\section{$\S 0$. Introduction.}

In this paper, general connections in the sense of $\mathrm{T}$. Otsuki are dealt with. The general connections were defined by $\mathrm{T}$. Otsuki in [O1] as a generalized notion of usual connections. Recently they are called Otsuki connections in Europe. He defined the general connections on the tangent tensor bundles of a manifold and defined associating geometrical objects analogous to those of usual ones, for example, their torsion forms and curvature forms. In his papers [O1]-[O11], several results about general connections were obtained. The purpose of this paper is to define general connections on vector bundles over a manifold and to study the fundamental properties.

In $\S 1$, we will prepare notations used in this paper and fundamental facts on the 1-jet bundle of a vector bundle. In $\S 2$, the general connections will be defined and some algebraic properties of the space of covariant derivatives of general connections will be studied. In $\S 3$, we will define two types of induced general connections which are induced by a pair of vector bundle homomorphisms and by a bundle map. In $\S 4$, using given general connections, we will construct general connections on the dual bundle and on the tensor product bundles. In $\S 5$, we will define the curvature form of a general connection.

The author would like to express his hearty thanks to Professor T. Otsuki for his helpful advice. He also would like to acknowledge the constant encouragement of Professor S. Yamaguchi.

\section{$\S 1$. Preliminaries.}

We assume that all objects are smooth and all vector bundles are real throughout this paper. Let $M$ be a manifold, $T(M)$ the tangent bundle and $C(M)$ the ring of real-valued functions on $M$. Let $V$ and $W$ be vector bundles over $M$. The fibre of $V$ at $p \in M$ will be denoted by $V_{p}$ and the dual bundle of $V$ is denoted by $V^{*}$. The space of cross-sections of $V$ will be denoted by $\Gamma(V)$. Let $\operatorname{HOM}(V, W)$ be the vector bundle of which fibre $\operatorname{HOM}(V, W)_{p}$ at $p$ is the vector space $\operatorname{HOM}\left(V_{p}, W_{p}\right)$ of linear maps from $V_{p}$ to $W_{p}$. Especially $\operatorname{HOM}(V, V)$ will be denoted by $\operatorname{END}(V)$. Note that $\operatorname{HOM}(V, W)$ can be naturally identified with the tensor product $V^{*} \otimes W$. The space of vector bundle homo-

Received October 2, 1984 
morphisms from $V$ to $W$ will be denoted by $\operatorname{HOM}(V, W)$. Especially $\operatorname{HOM}(V, V)$, the space of endomorphisms on $V$, will be denoted by $\operatorname{End}(V)$. Denote the identity (resp. zero) endomorphism of $V$ by $I_{V}$ (resp. $0_{V}$ ). Note that $\operatorname{HOM}(V, W)$ can be naturally identified with the space $\Gamma(H O M(V, W))$. We will generally use the same symbol to denote a vector bundle homomorphism and the induced linear map on the space of cross-sections.

For $s \in \Gamma(V)$, we will denote the 1 -jet of $s$ by $j^{1}(s)$ and the 1 -jet at $p$ by $j_{p}^{1}(s)$. Let $J^{1}(V)$ be the 1 -jet bundle of $V$.

DEFINITION. A vector bundle homomorphism $\iota: T(M)^{*} \otimes V \rightarrow I^{1}(V)$ is defined to be

$$
\iota\left((d f)_{p} \otimes s(p)\right):=j_{p}^{1}((f-f(p)) s) \quad \text { for } \quad f \in C(M), s \in \Gamma(V) .
$$

We have

LEMMA 1.1. $j^{1}(f s)=\iota((d f) \otimes s)+f j^{1}(s)$ for $f \in C(M), s \in \Gamma(V)$.

DEFINITION. A vector bundle homomorphism $\lambda: J^{1}(V) \rightarrow V$ is defined to be

$$
\lambda\left(j_{p}^{1}(s)\right):=s(p) \quad \text { for } \quad s \in \Gamma(V) .
$$

We have the following well-known fact:

LEMMA 1.2. (Jet bundle exact sequence)

$$
0 \longrightarrow T(M) * \otimes V \stackrel{\iota}{\longrightarrow} J^{1}(V) \stackrel{\lambda}{\longrightarrow} V \longrightarrow 0
$$

is:an exact sequence of vector bundles.

\section{$\S 2$. General connections.}

Let $V$ be a vector bundle over $M$.

DEFINITION. A vector bundle homomorphism $\gamma \in H O M\left(V, J^{1}(V)\right)$ is called a general connection on $V$. An endomorphism $P^{r}:=\lambda \circ \gamma \in E N D(V)$ is called the principal endomorphism of $\gamma$. A linear operator $\nabla^{r}: \Gamma(V) \rightarrow \Gamma\left(T(M)^{*} \otimes V\right)$, dedefined by

$$
\nabla^{r} s:=\iota^{-1}\left(j^{1}\left(P^{r} s\right)-\gamma(s)\right) \quad \text { for } s \in \Gamma(V),
$$

is called the covariant derivative of $\gamma$.

Note that $\iota^{-1}$ can be defined for $j^{1}\left(P^{\gamma} s\right)-\gamma(s)$ because of the exactness of the sequence in Lemma 1.2.

Remark. We can prove that the second order cotangent bundle appeared in [O1] is isomorphic with $J^{1}\left(T(M)^{*}\right)$. Thus our definition of a general connection coincides with that of $\mathrm{T}$. Otsuki in the case where $V=T(M)^{*}$. 
THEOREM. If $\nabla r$ is the covariant derivative of a general connection $\gamma$ with the principal endomorphism $P^{r}$, then

$$
\nabla^{r} f s=(d f) \otimes P^{r} s+f \nabla^{r} s \quad \text { for } \quad f \in C(M), s \in \Gamma(V) .
$$

Proof. From Lemma 1.1, we have

$$
\begin{aligned}
\nabla^{\gamma} f s & =c^{-1}\left(e\left((d f) \otimes\left(P^{r} s\right)\right)+f j^{1}\left(P^{\gamma} s\right)-f \gamma(s)\right) \\
& =(d f) \otimes\left(P^{\gamma} s\right)+f \iota^{-1}\left(j^{1}\left(P^{\gamma} s\right)-\gamma(s)\right) .
\end{aligned}
$$

We consider the following operators:

Definition. For $P \in E N D(V), O(V ; P)$ is the set of linear operators on $\Gamma(V)$ into $\Gamma\left(T(M)^{*} \otimes V\right)$ satisfying $\left(^{*}\right)$ as $\nabla^{r}$ in the above theorem. Put $O\left(V^{\prime}:=\right.$ $\cup\{O(V ; P) \mid P \in E N D(V)\}$.

Thus the above theorem shows that $\nabla^{r} \in O\left(V ; P^{r}\right)$ for $r \in H O M\left(V, J^{1}(V)\right)$. Conversely we have

Theorem. If $\nabla \in O(V ; P)$ for $P \in E N D(V)$, then there exists a unique $\gamma \in$ $H O M\left(V, J^{1}(V)\right)$ such that $P^{r}=P$ and $\nabla^{r}=\nabla$.

Proof. Put $\gamma(s):=j^{1}(P s)-\iota(\nabla s)$ for $s \in \Gamma(V)$. Since $\gamma(f s)=f \gamma(s)$ for $f \in C(M)$, we have $r \in H O M\left(V, J^{1}(V)\right)$.

For special examples, we have

THEOREM. $O\left(V ; 0_{V}\right)=H O M\left(V, T(M)^{*} \otimes V\right)$.

THEOREM. $O\left(V ; I_{V}\right)$ is equal to the set of covariant dervatives of usual connections on $V$.

For the study of general connections, we will mainly consider $O(V ; P)$ and $O(V)$ in this paper. Take $\nabla \in O(V ; P)$.

Definition. Given $v \in T(M)_{p}$ and $p \in M$, we define a linear map $\nabla_{v}: \Gamma(V)$ $\rightarrow V_{p}$ by $\nabla_{v} s:=i_{v}(\nabla s)$ for $s \in \Gamma(V)$, where $i_{v}$ is the inner product operator. Similarly given $X \in \Gamma(T(M))$, we define a linear operator $\nabla_{X}: \Gamma(V) \rightarrow \Gamma(V)$ by $\left(\nabla_{X} s\right)(p)$ : $=\nabla_{X(p)}$ s. We call $\nabla_{X}$ the covariant derivative along $X$.

Then clearly we have

TheOREm. The map $\Gamma(T(M)) \times \Gamma(V) \exists(X, s) \mapsto \nabla_{X} s \in \Gamma(V)$ is multi-linear and satisfies

$$
\nabla_{f X} s=f \nabla_{X} s \quad \text { and } \quad \nabla_{X} f_{s}=(X f) P s+f \nabla_{X} s \quad \text { for } f \in C(M) \text {. }
$$

Note that $\nabla_{v}$ unipuiquely determines the covariant derivative. The above theorems on general connections are analogous to those on usual connections. Moreover we can consider, in $O(V)$, the addition and the multiplication by 
elements of $E N D\left(V^{i}\right)$ as follows. Take two covariant derivatives $\nabla^{i} \in O\left(V ; P^{i}\right)$ $(i=1,2)$.

Definition. We define the sum $\nabla^{1}+\nabla^{2}$ by

$$
\left(\nabla^{1}+\nabla^{2}\right)_{X} s:=\nabla_{X}^{1} s+\nabla_{X}^{2} s \quad \text { for } s \in \Gamma(V), X \in \Gamma(T(M)) .
$$

For $Q \in E N D(V)$, we define the products $Q \nabla$ and $\nabla Q$ by

$$
(Q \nabla)_{X} s:=Q\left(\nabla_{X} s\right) \text { and }(\nabla Q)_{X} s:=\nabla_{X}(Q s) \text {. }
$$

Theorem. $\nabla^{1}+\nabla^{2} \in O\left(V ; P^{1}+P^{2}\right), Q \nabla \in O(V ; Q P)$ and $\nabla Q \in O(V ; P Q)$.

When $P$ has the inverse, we have

Corollary. If $Q P=P Q=I_{V}$, then $Q \nabla, \nabla Q \in O\left(V ; I_{V}\right)$.

TheOREM. The set $O(V)$ has a structure of $E N D(V)$-module with respect to the addition and multiplication defined as in the above definition.

Remark. In the case of the tangent bundle, the multiplication was defined and the associative law was stated in [O7].

\section{§3. Induced general connections.}

We will consider two types of induced general connections. The first type induced general connections are induced by a pair of vector bundle homomorphisms. The second type induced general connections are induced by a bundle map. The second type ones are analogous to the usual induced connections. The situation in the first type ones is slightly different from that in the case of usual connections as follows.

Let $V$ and $W$ be vector bundles over $M$. Take $L \in H O M(V, W), R \in H O M(W, V)$ and $\nabla \in O(V ; P)$.

Definition. A linear map $L \nabla R: \Gamma(W) \rightarrow \Gamma\left(T(M)^{*} \otimes W\right)$ is defined by

$$
(L \nabla R)_{X} t:=L\left(\nabla_{X}(R t)\right) \quad \text { for } \quad t \in \Gamma(W), X \in \Gamma(T(M)) \text {. }
$$

THEOREM. $\quad L \nabla R \in O(W ; L P R)$.

We will call the general connection determined by $L \nabla R$ the induced general connection induced by vector bundle homomorphisms. In the case of usual connections, we have

COROLlaRY. For a usual connection on $V$, the induced general connection on $W$ is a usual connection if and only if $L R=I_{W}$. 
When $R$ and $L$ satisfies $L R=I_{W}$ and $R L=I_{V}, V$ and $W$ are isomorphic as vector bundles and $O(V)$ and $O(W)$ are isomorphic as modules. If $V$ and $W$ are naturally isomorphic, then we will denote the corresponding covariant derivatives by the same symbol.

Remark. In the case where $V=T(M)$ and $W$ is a subbundle of $T(M)$, this type of induced general connections were essentially defined in [O7] and [O10].

Next we will consider the second type induced general connections. Let $N$ be a manifold and $F: N \rightarrow M$ a map. For a vector bundle $V$ over $M$, we denote the induced bundle over $N$ by $F^{\#} V$, the bundle map by $F: F^{\#} V \rightarrow V$ and its restriction to the fibre by $F_{q}:\left(F^{\#} V\right)_{q} \rightarrow V_{F(q)}$ for $q \in N$. Define $F^{\#}: \Gamma(V) \rightarrow \Gamma\left(F^{\#} V\right)$ by $\left(F^{\#} s\right)(q):=F_{q}^{-1}(s(F(q)))$ for $s \in \Gamma(V), \quad q \in N$. For $P \in E N D(V)$, define $F^{\#} P \in$ $E N D\left(F^{\#} V\right)$ by requiring $\left(F^{\#} P\right) F^{\#} s=F^{\#}(P s)$ for $s \in \Gamma(V)$.

THEOREM. For $\nabla \in O(V ; P)$, there exists a unique general connection $F^{\#} \nabla \in$ $O\left(F^{\#} V ; F^{\#} P\right)$ such that

$$
\left(F^{\# \nabla}\right)_{w} F^{\#} s=F_{q}^{-1}\left(\nabla_{F * w} s\right) \quad \text { for } \quad s \in \Gamma(V), w \in T(N)_{q}, q \in N,
$$

where $F_{*}$ is the differential of the map $F: N \rightarrow M$.

The proof of the theorem is analogous to that of the theorem on usual connections. We will call the general connection determined by $F^{\#} \nabla$ the induced general connection induced by a bundle map.

Remark. These two types of induced general connections can be applied to the study of submanifolds in spaces with general connections, for example, see $[\mathrm{O} 10],[\mathrm{H}]$ and $[\mathrm{OH}]$. In these papers, they used the induced general connections on the tangent bundles. Using also the induced general connection on the normal bundle, $\mathrm{H}$. Nemoto is studying the geometry of the submanifolds.

\section{$\S 4$. General connections on tensor product bundles.}

Let $V$ be a vector bundle over $M$. For $P \in E N D(V)$, define $P^{*} \in E N D\left(V^{*}\right)$ by $\left(P^{*} \eta\right)(s):=\eta(P s)$ for $\eta \in \Gamma\left(V^{*}\right), s \in \Gamma(V)$. Take a covariant derivative $\nabla \in O(V ; P)$.

Definition. A linear operator $\nabla^{*}: \Gamma\left(V^{*}\right) \rightarrow \Gamma\left(T(M)^{*} \otimes V^{*}\right)$ is defined by

$$
\left(\nabla_{X}^{*} \eta\right)(s):=X(\eta(P s))-\eta\left(\nabla_{X} s\right)
$$

for $\eta \in \Gamma\left(V^{*}\right), X \in \Gamma(T(M)), s \in \Gamma(V)$.

Note that $\nabla_{X}^{*} \eta \in \Gamma\left(V^{*}\right)$. Then we see

THEOREM. $\quad \nabla^{*} \in O\left(V^{*} ; P^{*}\right)$ for $\quad \nabla \in O(V ; P)$.

Let $V^{i}$ be vector bundles over $M$ and $P^{i} \in E N D\left(V^{i}\right)(i=1,2)$. Define $P^{2} \otimes P^{1}$ 
$\in E N D\left(V^{1} \otimes V^{2}\right)$ by requiring $\left(P^{1} \otimes P^{2}\right)\left(s_{1} \otimes s_{2}\right)=\left(P^{1} s_{1}\right) \otimes\left(P^{2} s_{2}\right)$ for $s_{i} \in \Gamma\left(V^{2}\right)$. Take $\nabla^{i} \in O\left(V^{i} ; P^{i}\right)(i=1,2)$.

DEFINITION. We define a linear operator $\nabla^{1} \otimes \nabla^{2}: \Gamma\left(V^{1} \otimes V^{2}\right) \rightarrow \Gamma\left(T(M)^{*} \otimes\right.$ $\left.\left(V^{1} \otimes V^{2}\right)\right)$ by requiring

$$
\left(\nabla^{1} \otimes \nabla^{2}\right)_{X}\left(s_{1} \otimes s_{2}\right)=\left(\nabla_{X}^{1} s_{1}\right) \otimes\left(P^{2} s_{2}\right)+\left(P^{1} s_{1}\right) \otimes\left(\nabla_{X}^{2} s_{2}\right)
$$

for $s_{i} \in \Gamma\left(V^{i}\right), X \in \Gamma(T(M))$.

THEOREM. $\quad \nabla^{1} \otimes \nabla^{2} \in O\left(V^{1} \otimes V^{2} ; P^{1} \otimes P^{2}\right)$ for $\nabla^{i} \in O\left(V^{i} ; P^{i}\right)(i=1,2)$.

As in the preceeding section, we will denote the corresponding covariant derivatives by the same symbol in a natural isomorphism, for example, $V^{1} \otimes V^{2}$ $\cong V^{2} \otimes V^{1}, \quad\left(V^{1} \otimes V^{2}\right) \otimes V^{3} \cong V^{1} \otimes\left(V^{2} \otimes V^{3}\right), \quad V^{*} \otimes W \cong H O M(V, W)$ and $V^{*} \otimes V^{*} \cong$ $(V \otimes V)^{*}$.

Denote $\left(\otimes^{r} V\right) \otimes\left(\otimes^{s} V^{*}\right)$ by $V^{(r, s)}$ and $\left(\otimes^{r} P\right) \otimes\left(\otimes^{s} P^{*}\right) \in E N D\left(V^{(r, s)}\right)$ by $P^{(r, s)}$ for $P \in E N D(V)$. We can define $\left(\otimes^{r} \nabla\right) \otimes\left(\otimes^{s} \nabla^{*}\right) \in O\left(V^{(r, s)} ; P^{(r, s)}\right)$. We denote this covariant derivative by $\nabla^{(r, s)}$ or simply by $\nabla$. From the definitions, we have

THEOREM. The covariant derivative $\nabla \in O\left(V^{(r, s)} ; P^{(r, s)}\right)$ satisfies

$$
\begin{aligned}
\nabla_{X}\left(s_{1} \otimes \cdots \otimes s_{r} \otimes \eta_{1} \otimes \cdots \otimes \eta_{s}\right)= & \sum_{i=1}^{r}\left(P s_{1}\right) \otimes \cdots \otimes\left(\nabla_{X} s_{\imath}\right) \otimes \cdots \otimes\left(P^{*} \eta_{1}\right) \otimes \cdots \otimes\left(P^{*} \eta_{s}\right) \\
& +\sum_{j=1}^{s}\left(P s_{1}\right) \otimes \cdots \otimes\left(P s_{r}\right) \otimes \cdots \otimes\left(\nabla_{X}^{*} \eta_{j}\right) \otimes \cdots \otimes\left(P^{*} \eta_{s}\right)
\end{aligned}
$$

for $s_{i} \in \Gamma(V), \eta_{j} \in \Gamma\left(V^{*}\right), \quad X \in \Gamma(T(M))$.

For future use, we prepare

THEOREM. The corresponding $\nabla \in O\left(E N D(V) ; P^{(1,1)}\right)$ satısfies

$$
\left(\nabla_{X} T\right) s=\nabla_{X}(T P s)-P T\left(\nabla_{X} s\right)
$$

for $T \in \Gamma(E N D(V)), s \in \Gamma(V), X \in \Gamma(T(M))$.

In contrast to the case of usual connections, $\nabla I_{V}$ does not vanish in general. We have

COROLLARY. $\quad\left(\nabla_{X} I_{V}\right) s=\nabla_{X}(P s)-P\left(\nabla_{X} s\right)$

THEOREM. The corresponding $\nabla \in O\left((V \otimes V)^{*} ; P^{(0,2)}\right)$ satısfies

$$
\left(\nabla_{X} g\right)\left(s_{1}, s_{2}\right)=X\left(g\left(P s_{1}, P s_{2}\right)\right)-g\left(\nabla_{X} s_{1}, P s_{2}\right)-g\left(P s_{1}, \nabla_{X} s_{2}\right)
$$

for $g \in \Gamma\left((V \otimes V)^{*}\right), s_{i} \in \Gamma(V)$. 


\section{$\S 5$. Curvature form of a general connection.}

Let $V$ be a vector bundle over $M$ and $\nabla \in O(V ; P)$ for $P \in E N D(V)$.

Definition. For $X, Y \in \Gamma(T(M))$, a map $K(\nabla)_{X, Y}$ on $\Gamma(V)$ is defined by

$$
K(\nabla)_{X, Y} s:=\nabla_{X}\left(\nabla_{Y}(P s)\right)-\nabla_{Y}\left(\nabla_{X}(P s)\right)-P\left(\nabla_{[X, Y]}(P s)\right)-\left(\nabla_{X} I_{V}\right) \nabla_{Y} s+\left(\nabla_{Y} I_{V}\right) \nabla_{X} s
$$

for $s \in \Gamma(V)$.

TheOREM. $K(\nabla) \in H O M\left(\Lambda^{2}(T(M)), E N D(V)\right)$.

Proof. Clearly $K(\nabla)_{X, Y}=-K(\nabla)_{Y, X}$. After straightforward but long calculations, we have

$$
K(\nabla)_{f X, Y} s=f K(\nabla)_{X, Y} s \quad \text { and } \quad K(\nabla)_{X, Y} f s=f K(\nabla)_{X, Y} s
$$

for $f \in C(M), s \in \Gamma(V)$.

We will call $K(\nabla)$ the curvature form of $\nabla$.

THEOREM. $K(Q \nabla) Q=Q K(\nabla Q)$ for $Q \in E N D(V)$.

Proof. From the definitions of $Q \nabla$ and $\nabla Q$, we have

$$
\left((Q \nabla)_{X} I_{V}\right) Q=Q\left((\nabla Q)_{X} I_{V}\right) \quad \text { for } \quad X \in \Gamma(T(M)) .
$$

Remark. $K(\nabla)$ coincides with the curvature form defined by $\mathrm{T}$. Otsuki in the case where $V=T(M)$. In [O2], he also defined the torsion form of a general connection on the tangent bundle.

\section{REFERENCES}

[H] Hour, C.S., Submanifolds in a Riemannian manifold with general connections, Math. J. Okayama Univ. 12 (1963), 1-37.

[NA] NAGAYAMA, H., A theory of general relativity by general connections I, TRU Math. 20 (1984), 173-187.

[NE] Nemoto, H., On submanifolds in spaces with general connections, preprint.

[O1] OTsukI, T., Tangent bundles of order 2 and general connections, Math. J. Okayama Univ. 8 (1958), 143-180.

[O2] OtsuKi, T., On general connections I, Math. J. Okayama Univ. 9 (1960), 99-164.

[O3] Otsuki, T., On general connections II, Math. J. Okayama Univ. 10 (1961), 113124.

[04] Otsuki, T., On normal connections, Kodai Math. Sem. Rep. 13 (1961), 152-166.

[O5] Otsuki, T., On metric general connections, Proc. Japan Acad. 37 (1962), 183-188.

[06] Otsuki, T., A note on metric general connections, Proc. Japan Acad. 38 (1962), 509-513.

[07] Otsuki, T., General connections $A \Gamma A$ and the parallelism of Levi-Civita, Kodai Math. Sem. Rep. 14 (1962), 40-52. 
[O8] Otsuki, T., On basic curves in spaces with normal general connections, Kodai Math. Sem. Rep. 14 (1962), 110-118.

[O9] OTsuki, T., On curvatures of spaces with normal general connections I, Kodai Math. Sem. Rep. 15 (1963), 52-61.

[010] OTsukI, T., On curvatures of spaces with normal general connections II, Kodai Math. Sem. Rep. 15 (1963), 184-194.

[O11] Otsuki, T., A note on general connections, Proc. Japan Acad. 41 (1965), 6-10.

[OH] Otsuki, T. And Houn, C.S., Ricci's formula for normal general connections and its applications, Kodai Math. Sem. Rep. 15 (1963), 184-194.

Department of Mathematics

FACUlTy OF SCIENCE

SCIENCE UNIVERsity OF TOKYO

TOKYO, JAPAN 162 